\title{
Ecología política: hacia un mejor entendimiento de los problemas socioterritoriales
}

\section{Political ecology: towards a better understanding of socio-territorial problems}

\author{
Robbins, Paul (20 i2), Political ecology: a CRitical introductions \\ to geOgRaphy, Second edition, Wiley-Blackwell, West Sussex, \\ 288 PP., ISBN: 978-0-470-65732-4.
}

La ecología política se ha convertido en uno de los marcos analíticos más utilizados para el análisis de los problemas socio-territoriales. Una amplia gama de disciplinas que van desde la geografía hasta los estudios de ciencia política, sociología y antropología han utilizado los preceptos básicos del estudio de este concepto; debido a la amplia gama de postulados teóricos y metodológicos que enmarca, la ecología política se ha consolidado como una herramienta teórica analítica que busca una mejor comprensión de los problemas dicotómicos entre hombre y ambiente.

Trabajos académicos relacionados con teorías de movimientos sociales, feminismo, y otras posiciones críticas sobre los procesos ambientales, han provisto las bases teóricas para el desarrollo de la ecología política como un marco que permite entender los procesos ambientales actuales y sus causalidades (Blaikie, 2008). Desde sus inicios, los trabajos académicos enmarcados dentro de la ecología política han retado la idea determinista dominante de que los problemas ambientales y la degradación de los recursos naturales están íntimamente vinculados con la pobreza, hambre y marginación de los grupos humanos, y que los recursos naturales están destinados a su sobreexplotación y destrucción debido al uso racional de los mismos (Hardin, 1968).

En este sentido, académicos como Piers Blaikie (2006), Nancy Lee Peluso (1993), Arturo Escobar (2008), Richard Peet y Michael Watts (1996), así como Paul Robbins (2004; 2012), han utilizado casos de estudio para ilustrar los principales conflictos que surgen del acceso, utilización, sobreexplotación, apropiación y manejo de los recursos naturales disponibles. Usan como base teórica la definición propuesta por Blaikie y Brookfield (1987: 17) "La ecología política combina las inquietudes de la ecología (antropología ecológica), y una economía política ampliamente definida. Juntos abarcan la dialéctica constante del cambio entre la sociedad y los recursos naturales, y también entre las clases y los grupos 
dentro de la sociedad misma”. La ecología política es un campo académico que busca criticar y caracterizar los fundamentos de la injusticia ambiental y la sobreexplotación de los recursos, su discusión parte de la idea de que el cambio ambiental está íntimamente correlacionado con procesos sociales y políticos a diversas escalas, y que para entender mejor cualquier problema ambiental es necesario vincular su análisis con las relaciones sociales de producción y la distribución del poder.

En este contexto, el libro que reseñamos representa uno de los más reconocidos aportes en el campo de la ecología política y de los estudios geográficos. En esta segunda edición del, ya ampliamente reconocido, libro se introducen los conceptos centrales, los principales exponentes, y los trabajos más relevantes en el extenso campo de la ecología política. Por medio de casos empíricos explicados concisa y claramente se exploran los argumentos clave y los retos explicativos contemporáneos que enfrenta la disciplina.

El libro describe la primera historia completa del desarrollo de la ecología política durante el último siglo, así como sus postulados teóricos; considera los retos más importantes que el campo de la ecología política enfrenta en la actualidad y en un futuro; estos retos se ilustran con casos de estudio de conflictos ambientales recientes, tal como el derrame petrolero en el golfo de México, asimismo se integran ilustraciones empíricas de ambientes, rurales y urbanos, provenientes de los países desarrollados y en vías de desarrollo.

Una de las principales críticas que ha recibido el campo de la ecología política tiene que ver con la definición de lo político dentro de los procesos ambientales. El autor contribuye a dicha discusión partiendo de la pregunta central: ¿qué es político en la ecología? Para responder a la interrogante, cuestiona los postulados malthusianos sobre la población y los recursos, los cuales consideran que a medida que la población mundial crece en proporción a la capacidad del sistema ambiental para soportarlos, existirán problemas sociales relacionados con hambrunas, enfermedades e incremento en la mortalidad; mientras que la naturaleza enfrentará problemas relacionados con recursos sobreexplotados que rebasarán el punto de auto-renovación.

El autor indica que todos los procesos de conflicto y degradación ambiental tienen un componente político; menciona que la ecología política presenta una alternativa para entender mejor dichos procesos, ya que, más que buscar los síntomas de los problemas, es necesario buscar y entender mejor sus causas, sobre todo en aquellas condiciones perniciosas donde los actores sociales explotan a otras personas y a los ambientes para generar ganancias a costo de la colectividad (p. 20). Además, indica que los sistemas ecológicos no son solamente políticos, sino también las ideas sobre los 
mismos, las cuales también se delimitan y definen a través de procesos económicos y políticos. Para ilustrar este punto, Robbins establece las cinco narrativas dominantes en ecología política: La degradación y marginación, la conservación y control de los recursos, el conflicto ambiental y la exclusión, los sujetos e identidades ambientales, y los actores y objetos políticos. En el libro estas cinco narrativas se ejemplifican en casos de estudio con información empírica; sin embargo, las cinco narrativas también ilustran las herramientas de teoría crítica general que la ecología política utiliza como fundamento. En la primera parte, el autor señala los orígenes teóricos de la disciplina, desde la economía política marxista y teoría de la propiedad común, hasta los postulados de gobernabilidad y gobernanza ambiental, así como la creación de sujetos ambientales, pasando por historia crítica ambientalista, estudios post-coloniales y teorías de poder y conocimiento. Todas estas disciplinas y postulados son analizados en esta sección en torno a las aportaciones teóricas que, en cada uno de sus campos, contribuyen a la ecología política.

Una de las aportaciones más importantes de esta obra es enmarcar el campo de la ecología política dentro de las corrientes teóricas críticas que permiten entender mejor los procesos dicotómicos entre ambiente y sociedad. En palabras del autor, no se busca crear una nueva teoría u ofrecer un método de investigación en particular, sino exponer un nuevo paradigma que permita plantear nuevas preguntas en torno a los procesos ambientales y sociales, así como uno que permita encontrar alternativas de explicación de los procesos actuales asociados con la devastación y sobreexplotación de los recursos, y la proliferación de problemas ambientales globales tales como el cambio climático, la erosión del suelo y los riesgos asociados a estos cambios.

El ejercicio académico, por tratar de encontrar nuevas formas para entender los procesos ambientales, no es exclusivo de la ecología política; En su texto, Robbins menciona que en la actualidad existe un dinamismo sin precedentes por parte de la comunidad académica internacional por encontrar nuevas formas para describir los procesos políticos y ambientales (p. 82), y es en este mismo ejercicio que se ha puesto a disposición una amplia gama de conceptos y nuevas formas para explorar los problemas ambientales. Sin embargo, en esta amplia variedad de explicaciones teóricas y analíticas radica una de las principales críticas hacia el campo de la ecología política. El mismo Piers Blaikie (2008) ha mencionado que parece ser más fácil enmarcar cualquier tema ambiental o de desarrollo dentro del campo de la ecología política, que dejarlo fuera. Debido a su amplia heterogeneidad y capacidad para adoptar aproximaciones teóricas y analíticas de otras disciplinas, se ha llegado a caracterizar a la ecología 
política como una "simple cubierta para el desarrollo anárquico" (Bryant, 1999: 148).

A pesar de esta gran diversidad, las cuestiones centrales de la ecología política continúan siendo abordadas por un conjunto de herramientas y procesos conceptuales comunes que son analizados por Robbins en este libro. Bajo la premisa de que la ecología política no es una teoría ni un método particular, sino más bien una comunidad en práctica, el autor explica que los análisis basados en ecología política requieren tomar en cuenta las posiciones políticas y los discursos de una amplia gama de actores. "La ecología política ha formado incuestionablemente una circunscripción general: se trata de una conversación a nivel global alrededor de un temario específico, uno que adopta una actitud crítica específica” (p. 87). Consecuentemente, una de las aportaciones de esta obra radica en caracterizar a la ecología política como una forma de representación, un argumento, o una aproximación al mejor entendimiento de los problemas dicotómicos entre el hombre y su entorno.

A pesar de la amplia gama y variedad de posiciones analíticas que convergen en el campo de la ecología política, una de sus principales características es su corte metodológico. En este sentido, Robbins menciona que uno de los problemas centrales de la investigación que relaciona el ambiente con la sociedad ha sido la necesidad de localizar sus problemas en un contexto local y regional. Para lograr este objetivo, la investigación basada en ecología política utiliza la denominada cadena de explicación para entender mejor no sólo las diferentes escalas en las que un problema socio-territorial se desarrolla, sino también las redes de actores que participan en cada una de éstas. La cadena de explicación, además de identificar los diferentes actores y sus discursos en torno a un problema socio-territorial, también permite identificar cuáles son las dinámicas económicas y políticas que tienen repercusiones en la degradación local de los recursos, esta es, sin duda alguna, una de las principales y más atractivas propuestas que ofrece la ecología política a los interesados en el estudio del ambiente y la sociedad.

En un esfuerzo por discutir los retos conceptuales y metodológicos que conlleva el uso de la ecología política, el autor utiliza una serie de casos de estudio para analizar los cambios y problemas ambientales. Dichos casos no solamente ilustran los principales problemas ambientales que aquejan al mundo en la actualidad, sino también los elementos conceptuales que los caracterizan, tal como la construcción social de los problemas y la naturaleza. El autor hace hincapié, no obstante, en que es necesario tener precaución metodológica y rigor para medir los cambios e imaginarios ambientales; de esto depende la relevancia de los análisis basados en ecología política. Una perspectiva compartida por uno de los precursores de 
la ecología política como campo del conocimiento, el mismo Piers Blaikie (2008) que pugna por una "ecología política que funcione", una ecología política que ofrezca nuevos horizontes en el entendimiento de los problemas ambientales que aquejan a la sociedad.

La segunda sección del libro, de acuerdo con el autor, busca complementar dos enfoques que los estudios tradicionales sobre medio ambiente y la sociedad han tratado como indisolubles; por un lado la destrucción y construcción de los sistemas y paisajes ambientales, y por el otro, las diferentes formas en las que ecología es un concepto construido socialmente por la humanidad a través de categorías y sistemas de conocimiento. En esta parte del libro, el autor enmarca las diferentes formas en la que ecologistas políticos han evaluado la destrucción de la naturaleza, el impacto humano sobre el ambiente y la degradación de los recursos de la tierra. Además, la discusión ilustra las serias tensiones que han existido entre las posiciones que definen los resultados ambientales no deseados como no-naturales (procesos que deben ser evitados y erradicados por ser considerados como problemas ambientales), y las posiciones que ven lo natural como estructuras o conceptos predominantemente sociales y culturales. En este apartado del libro el autor propone que las dos nociones dicotómicas deben ser suplantadas por una visión sintética de la producción ambiental, la cual toma de manera seria las implicaciones normativas de la degradación de la tierra, reconoce el carácter de construcción social de los aparatos conceptuales para entender la naturaleza, y es sensible a los componentes del sistema natural que participan en el cambio socio-ambiental.

Para cumplir con este objetivo, este apartado ilustra con casos empíricos que la destrucción o la construcción de los sistemas ambientales no es un proceso exclusivamente natural, también incluye procesos sociales, políticos y económicos que desempeńan un papel importante. Un aspecto fundamental de esta sección, es que, aunque el autor presenta evidencia empírica sólida para considerar que algunos procesos ambientales, más que naturales, son procesos políticos, culturales, económicos o incluso socialmente construidos, pareciera que la discusión metodológica al respecto pasa a un segundo plano, sobre todo considerando que en las secciones anteriores el autor hace un esfuerzo importante por describir los postulados y sobre todo las críticas que ha recibido la ecología política en términos metodológicos. A pesar de que la discusión se torna muy interesante al momento de que se abordan los principales retos explicativos que enfrenta la ecología política para dar un mejor entendimiento de los procesos de cambio ambiental y explicaciones causales, la discusión hubiera resultado enriquecida al tomar en consideración la forma en la que la cadena de explicación, como principal método de la ecología política, 
ayuda a encontrar los componentes de construcción social de los conflictos ambientales seleccionados. En otras palabras, explicar de una mejor manera la forma en la que la cadena de explicación coadyuva a entender mejor los retos y las causas de los problemas ambientales actuales.

La tercera parte del libro resulta de suma importancia para el lector interesado en temas de ecología política, pues se resaltan los discursos de los que toman decisiones, académicos, activistas o interesados en las cuestiones ambientales de actualidad, Robbins ofrece una entretenida discusión sobre las cinco narrativas ambientales más importantes de la actualidad. Los temas analizados son: la degradación y marginalización, conservación y control, conflicto ambiental, identidades y sujetos ambientales, y los actores y objetos políticos. Para cada una de estas discusiones se presenta un argumento, evidencia empírica que apoya dicho argumento, la evaluación del problema, y finalmente, cada narrativa se ilustra con casos de estudio actuales. Analizando estas cinco problemáticas ambientales, el autor sostiene que "hacer ecología política requiere paciencia, imaginación y disposición para fallar algunas veces" (p. 155), refiriéndose a la necesidad de estar abierto a la posibilidad de incluir puntos de vista incluso divergentes en el análisis de los problemas socio-territoriales.

De manera por demás interesante, el autor caracteriza los procesos de degradación y marginación a nivel mundial como un proceso vinculado con prácticas locales e inequidad en la distribución de los recursos. Con ilustraciones empíricas de casos en el bosque amazónico, la agricultura y la producción de bananas en el Caribe, y los problemas de propiedad de la tierra en el sudeste asiático; el autor analiza de manera clara cuáles son los patrones que originan procesos de marginación y degradación de los recursos. El tema de la conservación y control se caracteriza por los problemas que han surgido del cambio en la tendencia en patrones de producción global. Mientras, anteriormente, la producción de bienes de consumo se concentraba a nivel local e individual, actualmente la producción es colectiva; en este proceso de cambio, intereses globales y oficiales han deshabitado sistemas de modos de vida, de producción y organizaciones sociopolíticas locales. Esta problemática está ilustrada empíricamente por casos de sistemas pesqueros en Nueva Inglaterra, incendios forestales en Madagascar y la conservación social del bosque en Borneo.

De acuerdo con Robbins (p. 200):

La creciente escasez de los recursos producida por la apropiación de autoridades oficiales, corporaciones, o élites sociales, acelera los conflictos entre grupos en términos de género clase o etnicidad. De manera similar los problemas ambientales se politizan cuando grupos locales aseguran control de los recursos colectivos a costa de otros, acentuando (y legitimando) las intervenciones de manejo y desarrollo de los estados y las corporaciones. 
Los casos empíricos analizados en torno a esta situación son el desarrollo agrícola en Gambia y los conflictos de la tierra en el oeste americano.

"Las creencias y actitudes de la gente por sí mismas no conllevan a nuevas acciones, actitudes o sistemas de reglas ambientales; por el contrario, nuevas acciones, comportamientos y sistemas de reglas ambientales originan nuevos tipos de personas" (p. 216). Con esta afirmación Robbins asegura que los problemas ambientales además de crear vicisitudes para la sociedad, también originan nuevos sujetos ambientales. Los casos de la identidad y ecología maya en el sureste mexicano y la creación de sujetos ambientales en Estados Unidos ilustran esta aseveración en términos empíricos.

El autor asegura que los procesos de cambio ecológico y ambiental, a nivel global, tienen un componente político en todos sus casos. De esta forma la gente, instituciones, comunidades, corporaciones y naciones convergen y participan en nuevas redes de producción y consumo; reordenando las dinámicas de poder e influencia de la misma forma que los organismos y comunidades humanas lo hacen. La evidencia presentada por Robbins se sustenta en el análisis de la biotecnología agrícola y la conservación de osos en Norteamérica. Esta sección, en particular, coadyuva a difuminar dudas o cuestionamientos sobre lo que puede ser considerado como político en análisis de los problemas socio-territoriales.

En la última parte del libro, Robbins considera algunos de los límites del campo de la ecología política; especialmente su enfoque en casos discretos que pudieran ser calificados como pequeños en relación a sus conexiones a nivel mundial o a escalas globales. Por medio de la revisión de dos procesos ambientales diferentes, el autor ilustra la manera en la que la ecología política puede aportar argumentos que mejoren el análisis de procesos a escala global; el caso del cambio climático, y la resolución de procesos particulares a escala local, como es el caso de los jardines urbanos escolares.

Se puede concluir que el lenguaje claro y directo usado por Paul Robbins, en esta segunda edición de Political ecology..., es de particular importancia para los interesados en el campo de la ecología política y en su aplicación y uso para el análisis de los problemas dicotómicos entre hombre y sociedad. La información empírica y los ejemplos con los que ilustra cada uno de los conceptos teóricos, metodológicos y analíticos son importantes y de interés para académicos, estudiantes y personas relacionadas con los temas ambientales de la actualidad. En términos generales, el libro ofrece importantes aportaciones con respecto a los tres principios fundamentales de la ecología política; primero, que naturaleza es un concepto socialmente construido, donde la conservación y el manejo de los recursos naturales involucran una multiplicidad de actores con sus propias percep- 
ciones y perspectivas; segundo, que los problemas ambientales vinculados a comunidades locales no son exclusivamente un reflejo de las fallas en la implementación de políticas públicas, sino una manifestación de la influencia de fuerzas económicas y políticas externas, y tercero, que hay una necesidad urgente por cambiar radicalmente la forma en la que se concibe la economía política local y global como una manera para contribuir al mejor entendimiento de los problemas ambientales.

\section{Bibliografía}

Blaikie, Piers y H. Brookfield (1987), Land Degradation and Society, Methuen, London.

Blaikie, Piers (2006), "Is small really beautiful? Community-based natural resource management in Malawi and Botswana”, World Development, 34 (11), Elsevier Ltd., pp. 1942-1957.

Blaikie, Piers (2008), "Epilogue: towards a future for political ecology that works", Geoforum, 39 (2), Elsevier Ltd., pp. 765-772.

Bryant, R. L. (1999), "A political ecology for development countries? Progress and paradox in the evolution of a research field". Zeitschrift fur Wirtschaftsgeographie, 43 (3-4), Bad Soden, Buchenveri, pp. 148-157.

Escobar, Arturo (2008), Territories of difference: place, movements, life, redes, Duke University Press, London.

Hardin, G. (1968), “The tragedy of the commons", Science 162, 3859, American Association for the Advancement of Science, Washington DC, pp. 1234-1248.

Peet, Richard y Michael Watts (1996), Liberation ecologies, Routledge, Londres.

Peluso, Nancy Lee (1993), "Coercing conservation? The politics of state resource control”, Global Environmental Change 3 (2), Elsevier Ltd., pp. 199-217.

Robbins, Paul (2012), Political ecology: a critical introductions to geography, Wiley-Blackwell, West Sussex. 
Recibido: 27 de noviembre de 2012. Aceptado: 30 de enero de 2013.

Rafael Calderón-Contreras Universidad Autónoma Metropolitana, Unidad Cuajimalpa Correo-e: rcalderon@correo.cua.uam.mx

Rafael Calderón-Contreras. Es doctor en desarrollo internacional por la Universidad de East Anglia, Reino Unido, maestro en ciencias ambientales y desarrollo. Ha impartido cursos a nivel licenciatura y posgrado en México e Inglaterra. Cuenta con una amplia experiencia en análisis de gobernabilidad y acceso a recursos naturales, con interés particular en las prácticas y procesos que constituyen la propiedad de los recursos. Es profesor investigador del Departamento de Ciencias Sociales, división de Ciencias Sociales y Humanidades en la Universidad Autónoma Metropolitana, unidad Cuajimalpa. Sus intereses académicos y de investigación incluyen el acceso a recursos naturales, la propiedad de los recursos naturales, análisis socioterritoriales y de política ambiental, sistemas de información geográfica, riesgo, vulnerabilidad y resiliencia al cambio climático. Actualmente coordina proyectos de investigación sobre los retos y oportunidades del aprovechamiento de la biomasa lignocelulósica para la obtención de biocombustibles y otros productos de valor agregado en México y evaluación de la resiliencia de sistemas socioecológicos a los impactos del cambio climático global. Sus publicaciones recientes incluyen: "Between environmental policy and scientific knowledge: how might dryland environments challenge ideas regarding ecological dynamics", Ciencia Ergosum, Universidad Autónoma del Estado de México, Estado de México, 17 (1), pp. 81-87 (2010); Problematizing the role of community in natural resource management: the case of forest conservation in the Nevado de Toluca National Park, Lambert Academic Publishing, Germany (2010). 